\title{
Cushing disease-long-term outcome after transsphenoidal surgery
}

The long-term cure rate after transsphenoidal surgery for Cushing disease is $>70 \%$, new data reveal. The researchers of a retrospective cohort study, which included patients who were treated over a 20 -year period in a single center, also assessed resolution of clinical features and mortality after surgery, amongst other outcomes.

The investigators followed up 80 patients with Cushing disease who underwent transsphenoidal surgery between 1988 and 2009. The median age at diagnosis was 40 years, and the male-to-female ratio was 1.0:3.7. Hypertension (78\%), obesity (68\%) and osteoporosis (33\%) were common clinical features at diagnosis. The median clinical follow-up after surgery was 4.6 years, for 72 patients for whom data were available. The median follow-up for mortality, for all 80 patients, was 10.9 years.

Initial disease remission occurred in 60 of 72 (83\%), but disease recurrence occurred in eight individuals; the median time to recurrence was 2.1 years after surgery. The long-term outcomes were cure in 52 of 72 patients (72\%), persistent disease in 12 patients (17\%) and recurrent disease in eight patients (11\%).

Clinical improvements were apparent after successful transsphenoidal surgery. BMI reduced significantly between diagnosis and 6 weeks postoperatively and between 6 weeks and final follow-up. Rapid improvements in blood pressure were also observed after surgery in a number of patients.

Overall, 13 deaths occurred in the cohort; cardiovascular disease was the cause of eight of these deaths. The standardized mortality rate (SMR) was 3.17 (95\% CI 1.70-5.43). Excess mortality risk was nonsignificantly higher in patients with recurrent or persistent disease (SMR 4.12, 95\% CI 1.12-10.54) than in those who were cured (SMR 2.47, 95\% 0.80-5.77).

The researchers reflect that excess mortality still remains after effective

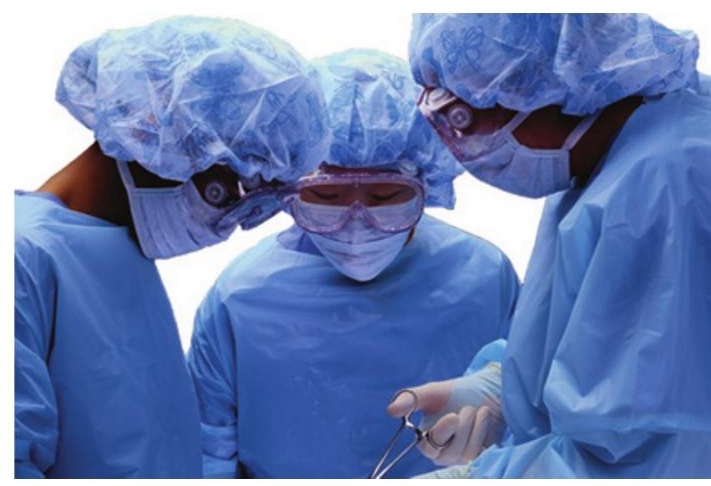

treatment for Cushing disease, which requires further investigation in large population-based studies.

\section{Carol Wilson}

Original article Hassan-Smith, Z. K. et al. Outcome of Cushing's disease following transsphenoidal surgery in a single center over 20 years. J. Clin. Endocrinol. Metab. doi:10.1210/jc.2011-2957 